Gynäkologe 2010 • 43:379-379

DOI 10.1007/s00129-009-2499-3

Online publiziert: 18. April 2010

๑) Springer-Verlag 2010

\author{
G. Emons ${ }^{1} \cdot$ M. Kupka ${ }^{2}$ \\ ${ }^{1}$ Klinik für Gynäkologie und Geburtshilfe, Georg-August-Universität Göttingen \\ ${ }^{2}$ Klinik und Poliklinik für Frauenheilkunde und Geburtshilfe, \\ Standort Innenstadt, Ludwig-Maximilians-Universität München
}

\title{
Dokumentationssysteme in der Frauenheilkunde
}

der Sprecher der Arbeitsgemeinschaft für Informationsverarbeitung in der Gynäkologie und Geburtshilfe (AIG) als Mitherausgeber dieses Themenheftes zur Verfügung. Gemeinsam konnten wir nun mit Sachkompetenz (M.K.) und kritischer Grundeinstellung (G.E.) an die Bearbeitung des Themas herangehen. onssysteme in der Gynäkologie“ gab, vermutete ich einen Irrtum. Ich protestierte, gab zu bedenken, dass ich über dieses Thema nie gearbeitet habe, bestenfalls Anwender sei, der unter den vielen Manifestationen der Dokumentationssysteme "leide“ und täglich seine Mitarbeiter motivieren müsse, ihren überbordenden Dokumentationspflichten nachzukommen. Der Senior Editor teilte mir lakonisch mit, dass ich genau aus diesem Grunde Herausgeber dieses Themenheftes geworden sei. Es solle nicht einmal mehr ein Loblied auf die Informationstechnologie (IT) und ihre großartigen Möglichkeiten gesungen, sondern eine kritische Diskussion über sinnvolle und nicht zielführende Dokumentationssysteme in unserem Fach angestoßen werden. Idealerweise könnten am Ende dieser Diskussion eine Optimierung der Dokumentationen und ihre Reduktion auf das Sinnvolle stehen und hoffentlich Mehrfachdokumentationen, das Anlegen von Datenfriedhöfen und ähnliche Fehlentwicklungen reduziert werden, die heute vielen Kollegen die Freude am Fach verderben und Ressourcen vernichten.

Die Darstellung der Kritik und des Unmutes bereitete mir (G.E.) keine Probleme, doch an der Sachkompetenz fehlte es mir. Dankenswerterweise stellte sich

\section{Ohne elektronische Systeme sind die Ansprüche an die Dokumentation nicht mehr zu befriedigen}

Wir konnten kompetente Autoren aus der AIG und außerhalb der Arbeitsgemeinschaft gewinnen, die in o. g. Sinne IT- und Dokumentationssysteme in unserem Fach, von E-Learning über Ultraschall-Teaching, EDV-Nutzung in der Praxis, der Reproduktionsmedizin, der Geburtshilfe und der gynäkologischen Onkologie, darstellen. Beim Studium der Beiträge wird schnell offenkundig, dass es ohne IT nicht mehr gehen wird. Die Ansprüche aus allen Bereichen an die Dokumentation in der Medizin und auch in unserem Fach sind derartig gewachsen, dass sie ohne elektronische Systeme nicht mehr zu befriedigen sind. Andererseits wird klar, dass in vielen Bereichen noch ein relevantes Optimierungspotenzial besteht, durch das insbesondere zermürbende Mehrfachdokumentationen vermieden werden müssen. Aber nicht nur die Software-Hersteller sind gefordert, sondern auch die Kolleginnen und Kollegen sowie die Herrschaften aus der Politik und auf Seiten der Kostenträger, die festlegen, was dokumentiert werden sollte. Auch hier ist eine Rückbesinnung auf das wirklich Wichtige und Zielführende indiziert.

Bei aller Kritik und allem Verbesserungsbedarf zeigen aber die Beiträge, welch wunderbares Potenzial in der Implementierung der IT in unser Fach steckt. Somit ist ein wenig Euphorie - zumindest Begeisterung - für Aspekte, bei denen der PC auf dem Schreibtisch auch umfangreichen Zugang zu Fachwissen und sekundenschnellen Meinungsaustausch um den Globus bedeuten können, nicht fehl am Platz.

Wir wünschen Ihnen viel Freude beim Lesen diese Ausgabe von „Der Gynäkologe" und hoffen, dass Sie sich in die kritisch konstruktive Weiterentwicklung der Dokumentationssysteme in unserem Fach einbringen werden.

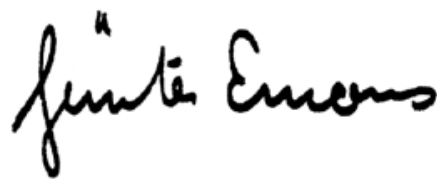

Prof. Dr. G. Emons

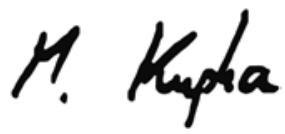

PD Dr. M.S. Kupka

\section{Korrespondenzadresse \\ Prof. Dr. G. Emons}

Klinik für Gynäkologie und Geburtshilfe, Georg-August-Universität Göttingen Robert-Koch-Str. 40, 37075 Göttingen emons@med.uni-goettingen.de 